\title{
Electromagnetic Structure of Charmed Baryons in Lattice QCD
}

\section{K. U. Can*, M. Oka}

Department of Physics, H-27, Tokyo Institute of Technology, Meguro, Tokyo 152-8551 Japan

E-mail: utku.can@th.phys.titech.ac.jp, oka@th.phys.titech.ac.jp

\section{G. Erkol, B.Isildak}

Department of Natural and Mathematical Sciences, Faculty of Engineering, Ozyegin University, Nisantepe Mah. Orman Sok. No:13, Alemdag 34794 Cekmekoy, Istanbul Turkey

E-mail: guray.erkol@ozyegin.edu.tr, bora.isildak@ozyegin.edu.tr

\section{T. T. Takahashi}

Gunma National College of Technology,

Maebashi, Gunma 371-8530, Japan

E-mail: ttoruenat.gunma-ct.ac.jp

The electromagnetic form factors of $\Sigma_{c}, \Xi_{c c}, \Omega_{c}$ and $\Omega_{c c}$ baryons are computed and their electric and magnetic charge radii as well as their magnetic moments are extracted in $2+1$ flavor Lattice QCD. Extrapolated physical point results show that the charge radii and magnetic moments are smaller compared to those of, e.g., proton. Investigating the individual quark contributions suggests that the existence of the heavy charm quark is responsible of such decrease.

The 32nd International Symposium on Lattice Field Theory,

23-28 June, 2014

Columbia University New York, NY

\footnotetext{
* Speaker.
} 


\section{Introduction}

The electric and magnetic properties of the hadrons can be probed by the electromagnetic form factors and valuable information can be extracted, such as their sizes or distributions of their components. The framework of lattice QCD enables us to determine such form factors starting from quark and gluon degrees of freedom. In our previous works we have found that the existence of a charm quark drives the charge radii and magnetic moments of the hadrons to smaller values $[1,2]$. Yet it is interesting to see how the charge radii are affected as the light quark gets heavier as in the case of replacing the $u / d$ quark by an $s$ quark.

In this work, report our results for the singly charmed $\Sigma_{c}^{(0,++)}(c d d, c u u), \Omega_{c}^{0}(c s s)$ baryons and the doubly charmed $\Xi_{c c}^{(+,++)}(c c d, c c u), \Omega_{c c}^{+}(c c s)$ baryons. In particular we compute the electromagnetic form factors and extract the electric and magnetic charge radii, and the magnetic moments of these baryons.

\section{Lattice Formulation and Setup}

Electromagnetic form factors can be calculated by considering the baryon matrix elements of the electromagnetic vector current $V_{\mu}=\sum_{q} e_{q} \bar{q}(x) \gamma_{\mu} q(x)$, where $q$ runs over the quark content of the given baryon. The matrix element can be written in the following form

$$
\left\langle B(p)\left|V_{\mu}\right| B\left(p^{\prime}\right)\right\rangle=\bar{u}(p)\left[\gamma_{\mu} F_{1, B}\left(q^{2}\right)+i \frac{\sigma_{\mu \nu} q^{v}}{2 m_{B}} F_{2, B}\left(q^{2}\right)\right] u(p),
$$

where $q_{\mu}=p_{\mu}^{\prime}-p_{\mu}$ is the transferred four-momentum. Here $u(p)$ denotes the Dirac spinor for the baryon with four-momentum $p^{\mu}$ and mass $m_{B}$. The Sachs form factors $F_{1, B}\left(q^{2}\right)$ and $F_{2, B}\left(q^{2}\right)$ are related to the electric and magnetic form factors by

$$
G_{E, B}\left(q^{2}\right)=F_{1, B}\left(q^{2}\right)+\frac{q^{2}}{4 m_{B}^{2}} F_{2, B}\left(q^{2}\right), \quad G_{M, B}\left(q^{2}\right)=F_{1, B}\left(q^{2}\right)+F_{2, B}\left(q^{2}\right) .
$$

Our method of computing the matrix element in Eq. (2.1), which was employed to extract the nucleon electromagnetic form factor, follows closely that of Ref.[3]. Using the following ratio

$$
\begin{aligned}
R\left(t_{2}, t_{1} ; \mathbf{p}^{\prime}, \mathbf{p} ; \Gamma ; \mu\right) & =\frac{\left\langle F^{B V_{\mu} B^{\prime}}\left(t_{2}, t_{1} ; \mathbf{p}^{\prime}, \mathbf{p} ; \Gamma\right)\right\rangle}{\left\langle F^{B B}\left(t_{2} ; \mathbf{p}^{\prime} ; \Gamma_{4}\right)\right\rangle} \\
& \times\left[\frac{\left\langle F^{B B}\left(t_{2}-t_{1} ; \mathbf{p} ; \Gamma_{4}\right)\right\rangle\left\langle F^{B B}\left(t_{1} ; \mathbf{p}^{\prime} ; \Gamma_{4}\right)\right\rangle\left\langle F^{B B}\left(t_{2} ; \mathbf{p}^{\prime} ; \Gamma_{4}\right)\right\rangle}{\left\langle F^{B B}\left(t_{2}-t_{1} ; \mathbf{p}^{\prime} ; \Gamma_{4}\right)\right\rangle\left\langle F^{B B}\left(t_{1} ; \mathbf{p} ; \Gamma_{4}\right)\right\rangle\left\langle F^{B B}\left(t_{2} ; \mathbf{p} ; \Gamma_{4}\right)\right\rangle}\right]^{1 / 2},
\end{aligned}
$$

where the baryonic two-point and three-point correlation functions are respectively defined as:

$$
\begin{aligned}
& \left\langle F^{B B}\left(t ; \mathbf{p} ; \Gamma_{4}\right)\right\rangle=\sum_{\mathbf{x}} e^{-i \mathbf{p} \cdot \mathbf{x}} \Gamma_{4}^{\alpha \alpha^{\prime}} \times\left\langle\operatorname{vac}\left|T\left[\eta_{B}^{\alpha}(x) \bar{\eta}_{B}^{\alpha^{\prime}}(0)\right]\right| \mathrm{vac}\right\rangle, \\
& \left\langle F^{B V_{\mu} B^{\prime}}\left(t_{2}, t_{1} ; \mathbf{p}^{\prime}, \mathbf{p} ; \Gamma\right)\right\rangle=-i \sum_{\mathbf{x}_{2}, \mathbf{x}_{\mathbf{1}}} e^{-i \mathbf{p} \cdot \mathbf{x}_{2}} e^{i \mathbf{q} \cdot \mathbf{x}_{1}} \Gamma^{\alpha \alpha^{\prime}}\left\langle\operatorname{vac}\left|T\left[\eta_{B}^{\alpha}\left(x_{2}\right) V_{\mu}\left(x_{1}\right) \bar{\eta}_{B^{\prime}}^{\alpha^{\prime}}(0)\right]\right| \mathrm{vac}\right\rangle,
\end{aligned}
$$

with $\Gamma_{i}=\gamma_{i} \gamma_{5} \Gamma_{4}$ and $\Gamma_{4} \equiv\left(1+\gamma_{4}\right) / 2$. The baryon interpolating fields are chosen, similarly to that of the octet baryons, as

$$
\begin{array}{cc}
\eta_{\Sigma_{c}}(x)=\varepsilon^{i j k}\left[\ell^{T i}(x) C \gamma_{5} c^{j}(x)\right] \ell^{k}(x), & \eta_{\Omega_{c}}(x)=\varepsilon^{i j k}\left[s^{T i}(x) C \gamma_{5} c^{j}(x)\right] s^{k}(x), \\
\eta_{\Xi_{c c}}(x)=\varepsilon^{i j k}\left[c^{T i}(x) C \gamma_{5} \ell^{j}(x)\right] c^{k}(x), & \eta_{\Omega_{c c}}(x)=\varepsilon^{i j k}\left[c^{T i}(x) C \gamma_{5} s^{j}(x)\right] c^{k}(x),
\end{array}
$$


where $\ell=u$ for the doubly charged $\Xi_{c c}^{++}(c c u) / \Sigma_{c}^{++}(c u u)$ and $\ell=d$ for the singly charged $\Xi_{c c}^{+}(c c d) / \Sigma_{c}^{+}(c d d)$ baryons. Here $i, j, k$ denote the color indices and $C=\gamma_{4} \gamma_{2} . t_{1}$ is the time when the external electromagnetic field interacts with a quark and $t_{2}$ is the time when the final baryon state is annihilated. When $t_{2}-t_{1}$ and $t_{1} \gg a$, the ratio in Eq. (2.3) reduces to the desired form

$$
R\left(t_{2}, t_{1} ; \mathbf{p}^{\prime}, \mathbf{p} ; \Gamma ; \mu\right) \underset{t_{2}-t_{1} \gg a}{\stackrel{t_{1} \gg a}{\longrightarrow}} \Pi\left(\mathbf{p}^{\prime}, \mathbf{p} ; \Gamma ; \mu\right) .
$$

We extract the form factors $G_{E, B}\left(q^{2}\right)$ and $G_{M, B}\left(q^{2}\right)$ by choosing appropriate combinations of Lorentz direction $\mu$ and projection matrices $\Gamma$ :

$$
\begin{aligned}
& \Pi\left(\mathbf{0},-\mathbf{q} ; \Gamma_{4} ; \mu=4\right)=\left[\frac{\left(E_{B}+m_{B}\right)}{2 E_{B}}\right]^{1 / 2} G_{E, B}\left(q^{2}\right), \\
& \Pi\left(\mathbf{0},-\mathbf{q} ; \Gamma_{j} ; \mu=i\right)=\left[\frac{1}{2 E_{B}\left(E_{B}+m_{B}\right)}\right]^{1 / 2} \varepsilon_{i j k} q_{k} G_{M, B}\left(q^{2}\right) .
\end{aligned}
$$

Here, $G_{E, B}(0)$ gives the electric charge of the baryon. Similarly, the magnetic moment can be obtained from the magnetic form factor $G_{M, B}$ at zero momentum transfer.

We run our simulations on $32^{3} \times 64,2+1$-flavor configurations generated by the PACS-CS Collaboration [4] using the Clover fermion action and the Iwasaki gauge action. We use the gauge configurations at $\beta=1.90$ with the Clover coefficient $c_{S W}=1.715$, which give a lattice spacing of $a=0.0907(13) \mathrm{fm}(a 1=2.176(31) \mathrm{GeV})$. The simulations are carried out with four different hopping parameters for the light sea and valence quarks, $\kappa_{u, d}^{\text {sea }}, \kappa_{u, d}^{\mathrm{val}}=0.13700,0.13727,0.13754$ and 0.13770, which correspond to pion masses of approximately 702, 570, 411, and $296 \mathrm{MeV}$. The strange quark mass is fixed to its physical value at $\kappa_{\text {sea }}^{s}=0.13640$. In order to be consistent with the sea quarks we use the clover action for the $\mathrm{u}, \mathrm{d}$ and $\mathrm{s}$ valence quark propagators and we take $\kappa_{\text {sea }}^{q}=\kappa_{\text {val }}^{q}$.

We employ a wall method [1], which provides a simultaneous study of all the hadrons we are considering. The source is Gaussian smeared in a gauge-invariant manner. The source-sink separation is chosen to be 12 lattice units in the temporal direction. In the case of $u, d$ and $s$ quarks, we choose the smearing parameters so as to give a root-mean-square radius of $\left\langle r_{l}\right\rangle \sim 0.5 \mathrm{fm}$. As for the charm quark, we adjust the smearing parameters to obtain $\left\langle r_{c}\right\rangle=\left\langle r_{l}\right\rangle / 3$.

For the charm quarks, we apply the Fermilab method [5] in the form employed by the Fermilab Lattice and MILC Collaborations [6, 7]. In this simplest form of the Fermilab method, the clover coefficients $c_{E}$ and $c_{B}$ in the action are set to the tadpole-improved value $1 / u_{0}^{3}$, where $u_{0}$ is the average link. Following the approach in Ref. [8], we estimate $u_{0}$ to be the fourth root of the average plaquette. We tuned the charm-quark hopping parameter to $\kappa_{c}=0.1246$ by comparing the experimental spin-averaged static masses of charmonium and heavy-light mesons with our lattice results [9].

For each $\kappa_{\text {sea }}^{u d}$ value, we perform our measurements on 100, 100, 150 and 170 different configurations for the $\Sigma_{c}$ and $\Xi_{c c}$ and 100,100,100 and 130 different configurations for the $\Omega_{c}$ and $\Omega_{c c}$ baryons. In order to increase the statistics we use multiple source-sink pairs for the $\Sigma_{c}$ and $\Xi_{c c}$ baryons. We insert momentum through the current up to nine units: $\left(\left|p_{x}\right|,\left|p_{y}\right|,\left|p_{z}\right|\right)=(0,0,0),(1,0,0)$, $(1,1,0),(1,1,1),(2,0,0),(2,1,0),(2,1,1),(2,2,0),(2,2,1)$ and average over equivalent momenta in the 

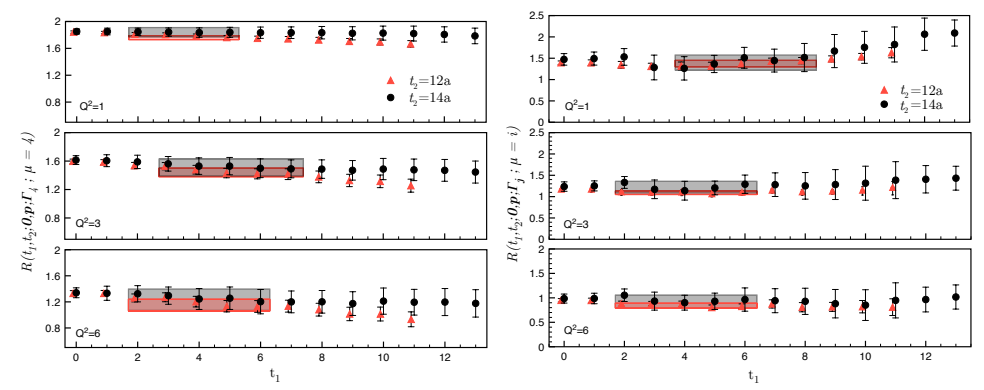

(a)

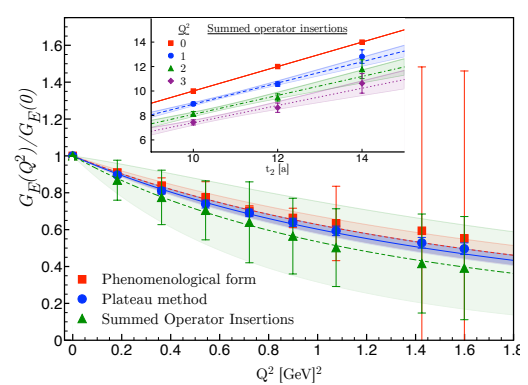

(b)

Figure 1: (a) The ratio in Eq. 2.3 as function of the current insertion time, $t_{1}$, for the electric (left) and magnetic (right) form factor of $\Xi_{c c}$ with $t_{2}=12 a$ and $t_{2}=14 a$. We show statistics over 30 configurations for three illustrative momentum-transfer values. The data for $t_{2}=12 a$ are slightly shifted to left for clear viewing. (b) A comparison of the electric form factor of $\Xi_{c c}$ for the heaviest quark mass, as obtained using a simple plateau fit, the phenomenological fit form and the summation method. The small panel depicts the summed operator insertions for three time separations and for the first four momentum insertions with their linear fits.

case of electric form factor. For the magnetic form factor we average over all equivalent combinations of spin projection, Lorentz component and momentum indices. We use the point-split lattice current, $V_{\mu}=1 / 2\left[\bar{q}(x+\mu) U_{\mu}^{\dagger}\left(1+\gamma_{\mu}\right) q(x)-\bar{q}(x) U_{\mu}\left(1-\gamma_{\mu}\right) q(x+\mu)\right]$, which is conserved by the Wilson fermions, therefore does not require any renormalisation. All statistical errors are estimated by the single-elimination jackknife analysis and the $\chi^{2}$ p-values are used to test the goodness of fits.

In our simulations, the source-sink time separation is fixed to $1.09 \mathrm{fm}\left(t_{2}=12 a\right)$. There are works in the literature for the nucleon axial and electromagnetic form factors $[3,10]$ as well as for the $\Omega^{-}$electromagnetic form factors [11] which finds a separation of $1 \mathrm{fm}$ between the source and sink is sufficient to avoid excited-state contaminations. To check that a separation of $t_{2}=12 a$ is sufficient for the charmed baryons, we compare our electric and magnetic results for the form factors of $\Xi_{c c}$ with $t_{2}=12 a$ and $t_{2}=14 a$ as shown in Fig. 1a. The plateau values obtained from the two time separations are consistent with each other, implying that the shorter source-sink time separation is sufficient. Other baryons we study exhibit a similar behaviour, therefore we use the shorter separation i.e. $t_{2}=12 a$ in all of our analysis.

To further ensure that the ground state baryon is isolated from the excited-state contaminations we performed a secondary analysis and fitted the ratio in Eq. (2.3) to a phenomenological form

$$
R\left(t_{2}, t_{1}\right)=G_{E, M}+b_{1} e^{-\Delta t_{1}}+b_{2} e^{-\Delta\left(t_{2}-t_{1}\right)},
$$

where $\Delta$ is the energy gap between the ground and the excited state. Since the energy gap is not known for charmed baryons we leave $\Delta$ as a free parameter together with $b_{1}$ and $b_{2}$, which yields a larger uncertainty for $G_{E, M}$.

In Fig. 1b we compare the electric form factor of $\Xi_{c c}$ as obtained using a simple plateau fit and the phenomenological fit form in Eq. (2.10), for the heaviest quark mass. The two fit forms give completely consistent results, with the phenomenological fit form having twice as large errors. 
We have not been able to obtain a good fit to the phenomenological form for the magnetic form factors. The statistical errors in the parameters of the fit to $R\left(t_{2}, t_{1}\right)$ are too large to allow a precise determination of $G_{M}$. Therefore, we use solely a plateau fit in extracting the ground state matrix elements of electric and magnetic form factors. We show all the plateau fits in Ref. [9].

We also consider the summed operator insertions method [12] with three source-sink separations, namely for $t_{2}=10 a, t_{2}=12 a$ and $t_{2}=14 a$ (for the heaviest quark mass and using 30 configurations). Since this is a computationally extensive method we have employed it as an exploratory case in this work. Fig. $1 \mathrm{~b}$ depicts a comparison of the summation method with the other methods also. In general, the data are consistent within the errors but the statistical errors for the summation method is still large. We intend to study the summation method further with increased statistics in a future work.

\section{Results and Discussion}

We use the dipole form to describe the $Q^{2}$ dependence of the baryon form factors:

$$
G_{E, M}\left(Q^{2}\right)=G_{E, M}(0) /\left(1+Q^{2} / \Lambda_{E, M}^{2}\right)^{2} .
$$

In Fig. 2 we only give the electric form factors of $\Sigma_{c}^{++}, \Xi_{c c}^{++}$and $\Omega_{c c}^{+}$, as normalized with their electric charges, to illustrate our fits. Fits on all different $\kappa_{u, d}$ lattices are shown in the figure. The magnetic form factors of $\Xi_{c c}^{+}, \Omega_{c c}^{+}, \Omega_{c}^{0}, \Sigma_{c}^{0}$ and $\Sigma_{c}^{++}$baryons can be found in Ref. [9].

We can extract the electric/magnetic charge radii of the baryons from the slope of the form factor at $Q^{2}=0$ by, $\left\langle r^{2}\right\rangle=-\left.\frac{6}{G(0)} \frac{d}{d Q^{2}} G\left(Q^{2}\right)\right|_{Q^{2}=0}$. Using the dipole form in Eq. (3.1), we get $\left\langle r_{E, M}^{2}\right\rangle=$ $12 / \Lambda_{E, M}^{2}$. Then the charge radii can be directly calculated using the values of dipole masses as obtained from our simulations.

The magnetic moment is defined as $\mu_{B}=G_{M}(0) e /\left(2 m_{B}\right)$ in natural units. We obtain $G_{M}(0)$ by extrapolating the lattice data to $Q^{2}=0$ via the dipole form in Eq. (3.1) as explained above. We evaluate the magnetic moments in nuclear magnetons using the relation $\mu_{B}=G_{M}(0)\left(e / 2 m_{B}\right)=$ $G_{M}(0)\left(m_{N} / m_{B}\right) \mu_{N}$, where $m_{N}$ is the physical nucleon mass and $m_{B}$ is the baryon mass as obtained on the lattice.
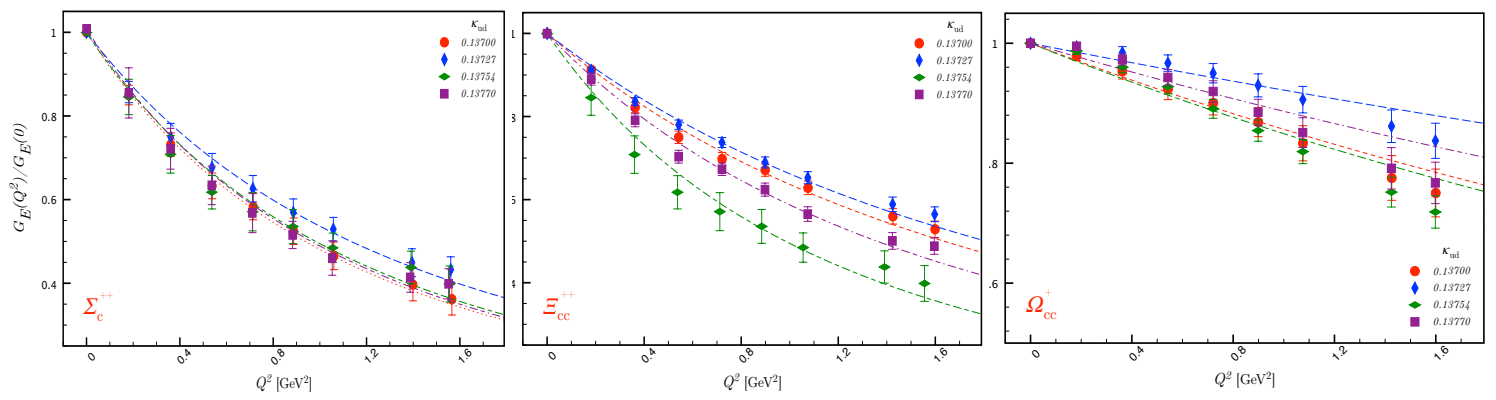

Figure 2: The electric form factors of $\Sigma_{c}^{++}, \Xi_{c c}^{++}$and $\Omega_{c c}^{+}$as normalized with their electric charges as functions of $Q^{2}$, for all the quark masses we consider. The dots mark the lattice data and the curves show the best fit to the dipole form in Eq. (3.1).

In Table 1, we give the electric and magnetic charge radii in $\mathrm{fm}^{2}$, and the magnetic moments $\left(\mu_{B}\right)$ in nuclear magnetons at the chiral point. These numerical values with their chiral extrapo- 
lations and results at each light quark kappa can be found in Ref. [9]. Some select chiral extrapolations are given in Fig. 3 for illustration. We used three fit forms that are constant, linear and quadratic in $m_{\pi}^{2}$ as, $f_{\text {con }}=c, f_{\text {lin }}=a m_{\pi}^{2}+b, f_{\text {quad }}=a m_{\pi}^{4}+b m_{\pi}^{2}+c$, where $a, b$ and $c$ are the fit parameters.

Table 1: Extrapolated results of charge radii and magnetic moments.

\begin{tabular}{c|ccccccc}
\hline \hline & $\left\langle r_{E, \Sigma_{c}^{++}}^{2}\right\rangle$ & $\left\langle r_{E, \Xi_{c c}^{++}}^{2}\right\rangle$ & $\left\langle r_{E, \Omega_{c c}^{+}}^{2}\right\rangle$ & $\left\langle r_{M, \Sigma_{c}^{++}}^{2}\right\rangle$ & $\left\langle r_{M, \Sigma_{c}^{0}}^{2}\right\rangle$ & $\left\langle r_{M, \Omega_{c}^{0}}^{2}\right\rangle$ & \\
\hline \hline Lin. Fit & $0.192(22)$ & $0.136(8)$ & $0.032(6)$ & $0.410(81)$ & $0.377(75)$ & $0.297(33)$ & \\
Quad. Fit & $0.234(37)$ & $0.165(12)$ & $0.043(11)$ & $0.696(153)$ & $0.650(126)$ & $0.354(54)$ & \\
\hline \hline & $\left\langle r_{M, \Xi_{c c}^{+}}^{2}\right\rangle$ & $\left\langle r_{M, \Omega_{c c}^{+}}^{2}\right\rangle$ & $\mu_{\Sigma_{c}^{++}}$ & $\mu_{\Sigma_{c}^{0}}$ & $\mu_{\Omega_{c}^{0}}$ & $\mu_{\Xi_{c c}^{+}}$ & $\mu_{\Omega_{c c}^{+}}$ \\
\hline \hline Lin. Fit & $0.135(10)$ & $0.135(11)$ & $1.569(253)$ & $-0.852(133)$ & $-0.608(45)$ & $0.411(15)$ & $0.405(13)$ \\
Quad. Fit & $0.154(19)$ & $0.148(21)$ & $2.220(505)$ & $-1.073(269)$ & $-0.639(88)$ & $0.425(29)$ & $0.413(24)$ \\
\hline \hline
\end{tabular}

Our results show that the doubly charged $\Sigma_{c}^{++}$has the largest electric charge radius amongst the baryons that we considered. The electric charge radii of $\Omega_{c c}^{+}$and $\Xi_{c c}^{+}$[2] are about the same size which suggests that electric charge radius is insensitive to replacing the light $d$-quark by an $s$-quark. We observe that the electric charge radii is much smaller compared to that of the proton (the PDG value is $\left\langle r_{E, p}^{2}\right\rangle=0.770 \mathrm{fm}^{2}$ [13]). The magnetic charge radii of $\Sigma_{c}^{++}$and $\Sigma_{c}^{0}$ are close to that of the proton's, which is $\left\langle r_{M, p}^{2}\right\rangle=0.604 \mathrm{fm}^{2}$ [13]. The $m_{\pi}^{2}$ dependence of the electric and magnetic charge radii's seems to be better modelled by a quadratic fit compared to the other fit models that we considered. A magnetic charge radii extrapolation plot is given in Figure 3a. The charge radii of the $\Omega_{c}$ and $\Omega_{c c}$ baryons, as apparent in Fig. $3 \mathrm{~b}$ for the electric charge radius case, show peculiar fluctuations with respect to the light-quark masses even though they don't contain valance light-quarks. Such behaviour remains as an open question. On the other hand, Fig. 3c shows that their magnetic moments are free from such behaviour and nicely modelled by a linear fit. We refer the reader to Ref. [9] for a more detailed discussion and all the plots.

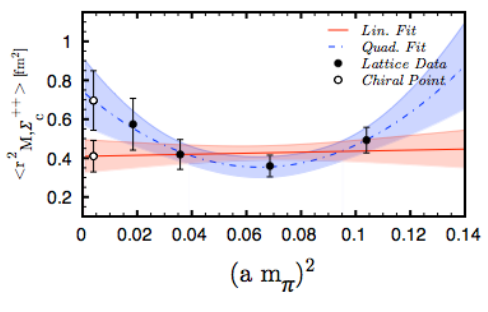

(a)

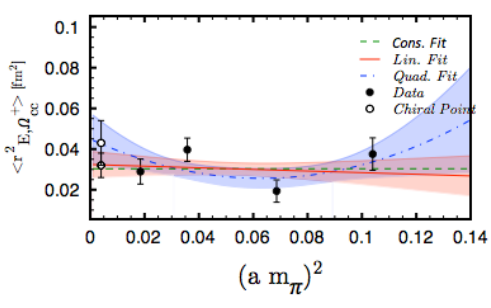

(b)

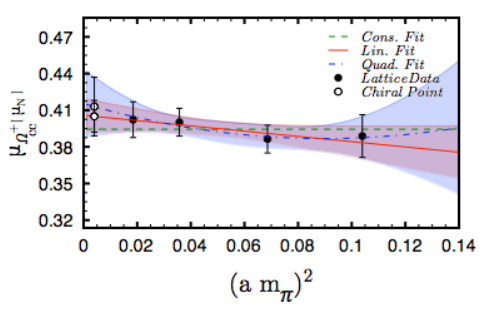

(c)

Figure 3: Select chiral extrapolations. a) Magnetic charge radii of $\Sigma_{c}^{++}$, b) Electric charge radii of $\Omega_{c c}^{+}$, c) magnetic moment of $\Omega_{c c}^{+}$. We show the fits to constant, linear and quadratic forms. The shaded regions are the maximally allowed error regions for the fit forms.

Analysing the individual quark contributions [9] we see that the the charm quark contributions are independent of the quark content of the baryons and the contributions from the $u / d$-and s-quark are roughly the same, thus leading $\Sigma_{c}^{++}$and $\Xi_{c c}^{++}$, as well as, $\Omega_{c c}^{+}$and $\Xi_{c c}^{+}$to have almost the same sizes. The small contribution of $c$ quark suggests that the centre of mass is shifted towards it and 
this causes the baryon to shrink even though the light quark contributions are systematically larger. The dominant contribution to the magnetic moments come from the doubly represented quarks and the opposite signs of the light and heavy quark contributions suggest that their spins are generally anti-aligned within the baryon. $\Sigma_{c}^{++}$has the largest magnetic moment of all and the strange baryons $\Omega_{c}$ and $\Omega_{c c}$ have somewhat smaller moments. It is interesting to compare these values with the experimental magnetic moment of the proton, which is $\mu_{p}=2.793 \mu_{N}$ [13]. Comparing our magnetic moment results with several other models we see a quantitative disagreement even though the signs match [9].

\section{Conclusions}

We have calculated the electromagnetic form factors of the $\Sigma_{c}, \Omega_{c}, \Xi_{c c}, \Omega_{c c}$ baryons and extracted their electric and magnetic charge radii and the magnetic moments from $2+1$-flavor simulations of QCD on a $32^{3} \times 64$ lattice. Our results imply that the charmed baryons are compact with respect to baryons that are composed of only light quarks, e.g., the proton. The existence of the heavy quark shrinks the baryons and doubly charmed baryons are more compact than the singly charmed baryons of the same charge. The magnetic moments are dominantly determined by the doubly represented quarks. The signs of the magnetic moments are correctly reproduced on the lattice. However, in general we see an underestimation of the magnetic moments as compared to what has been found with other theoretical methods. A work is underway on nearly physical-point ensembles.

The numerical calculations were performed on National Center for High Performance Computing of Turkey (Istanbul Technical University) under project number 10462009. We used a modified version of CHROMA [14]. This work is supported in part by The Scientific and Technological Research Council of Turkey (TUBITAK) under project number 110T245 and in part by KAKENHI under Contract Nos. 22105503, 24540294 and 22105508.

\section{References}

[1] K. U. Can, G. Erkol, M. Oka, A. Ozpineci and T. T Takahashi,Phys.Lett. B719, 103 (2013)

[2] K. U. Can, G. Erkol, B. Isildak, M. Oka and T. T Takahashi, Phys. Lett. B726, 703 (2013)

[3] C. Alexandrou, M. Brinet, J. Carbonell, M. Constantinou, P. Harraud, Phys.Rev. D83, 094502 (2011)

[4] S. Aoki et al. (PACS-CS Collaboration), Phys. Rev. D79, 034503 (2009)

[5] A. X. El-Khadra, A. S. Kronfeld and P. B. Mackenzie, Phys. Rev. D55, 3933-3957 (1997).

[6] T. Burch, C. DeTar, M. Di Pierro, A.X. El-Khadra, E. D. Freeland et al., Phys. Rev. D81, 034508 (2010).

[7] C. Bernard et al, Phys. Rev. D83, 034503 (2011).

[8] D. Mohler and R. M. Woloshyn, Phys. Rev. D83, 034503 (2011).

[9] K. U. Can, G. Erkol, B. Isildak, M. Oka and T. T Takahashi, J. High Energy Physics JHEP05(2014)125.

[10] C. Alexandrou et al., Phys. Rev. D83, 045010 (2011).

[11] C. Alexandrou, T. Korzec, G. Koutsou, J. W. Negele and Y. Proestos, Phys. Rev. D82, 034504 (2010).

[12] L. Maiani, G. Martinelli, M. L. Paciello and B. Taglienti, Nucl. Phys. B293, 420 (1987).

[13] J. Beringer et al. (Particle Data Group), Phys. Rev. D86, 010001 (2012).

[14] R. G. Edwards and B. Joo (SciDAC Collaboration, LHPC Collaboration, UKQCD Collaboration), Nucl.Phys.Proc.Suppl. 140, 832 (2005) 